\title{
IMPLEMENTING A DATA-DRIVEN SIMULATION METHOD FOR QUANTIFYING PIPE WELDING OPERATOR QUALITY PERFORMANCE
}

\author{
Wenying $\mathrm{Ji}^{1}$, and Simaan M. AbouRizk ${ }^{2}$
}

\begin{abstract}
This paper proposes a framework for implementing a Markov Chain Monte Carlo (MCMC)-based posterior distribution determination approach to quantify pipe welding operator quality performance for industrial construction projects. The existing quality management data and engineering design data from a pipe fabrication company are processed and analysed to demonstrate the feasibility and applicability of the proposed approach. Through the use of a specialised Metropolis-Hastings algorithm, operator welding performance is quantified and uncertainty is incorporated. Practitioners can utilize outputs of the proposed method to infer operator welding quality performance of a particular weld type and identify operators with exceptional quality performance. Potential applications of the research findings are discussed from the perspectives of production planning, employee training, and strategic recruiting.
\end{abstract}

Keywords: Industrial construction, pipe fabrication, quality management, fraction nonconforming, Markov Chain Monte Carlo (MCMC).

\section{INTRODUCTION}

Due to its ability to improve overall project performance, many industrial construction projects have implemented a modularized approach to construction. Essential to modularization is the process of pipe spool fabrication (Wang et al 2009). Pipe spools are primarily assembled by welding, which must be sampled and tested to ensure that specified welding quality requirements are satisfied (Ji and AbouRizk, 2016). Consequently, operators with exceptional quality performance may considerably enhance company efficiency and productivity, while poor quality performance may lead to quality-associated cost and schedule overruns.

However, due to the vast possible combinations of pipe attributes (e.g., nominal pipe size (NPS), pipe schedule, material), and consequently, weld type, it is often difficult for practitioners to accurately and reliably estimate operator performance from historical welding data. Furthermore, estimation of quality performance can be confounded by the inappropriate incorporation of sampling uncertainty, which arises when inspection data is collected from a representative sample rather than an entire population (Weiss, 2013).

Recently, Ji and AbouRizk (2017) have advocated for the implementation of a Markov Chain Monte Carlo (MCMC)-based numerical method to incorporate sampling uncertainty during welding quality performance analyses. Weld quality is often assessed by nondestructive examination (NDE), which detects discontinuities in welds without

$1 \quad$ Ph.D. Candidate, University of Alberta, Department of Civil \& Environmental Engineering, 9105116 St., 5-080 NREF, Edmonton, Alberta, Canada, T6G 2W2, wenying.ji@ualberta.ca

2 Professor, University of Alberta, Department of Civil \& Environmental Engineering, 9105116 St., 5080 NREF, Edmonton, Alberta, Canada, T6G 2W2, abourizk@ualberta.ca 
inducing pipe damage (ASME 2005). From this, a percentage repair rate, defined as the number of failed NDE over the number of NDE completed, can be calculated. This is consistent with the concept of fraction nonconforming in statistical quality control theory, which is defined as the ratio of nonconforming items to the total number of items in a population (Montgomery, 2007). The fraction nonconforming can be modelled as a binomial variable and follows a beta distribution (Ji and AbouRizk 2017).

The Markov Chain Monte Carlo (MCMC)-based numerical method proposed by Ji and AbouRizk (2017) calculates a posterior distribution that covers the true population fraction nonconforming of each pipe weld type. This method also has the advantage of deriving additional statistical values, such as mean, median, variance, and quantiles, which can be used to assess historical welding quality performance and improve decision-making processes (Weaver and Hamada 2016).

To facilitate the quantification of operators' welding quality performance, this study proposes the implementation of a data-driven simulation model that efficiently sources and classifies existing quality management and engineering design data, while concurrently incorporating sampling uncertainty. Specifically, the proposed framework (1) fuses and transforms data from separate data sources into interpretable data; (2) implements an MCMC-based approach to numerically estimate the posterior distribution of operators' welding quality performance; and (3) proposes potential applications to comprehensively improve pipe welding quality performance.

The remainder of this paper is organized as follows: In the next section, the framework of the proposed methodology, which includes data source identification, data adapter design, algorithm development, and a demonstration of the primary outputs, is described. In the subsequent section, potential applications of the research outcome from the perspectives of production planning, employee training, and strategic recruiting, will be discussed. Finally, conclusions regarding principle findings, contributions, and limitations of this research will be summarized.

\section{RESEARCH FRAMEWORK}

Here, a framework for implementing a data-driven simulation model for quantifying pipe welder quality performance is proposed. Framework functionality is demonstrated using data from a pipe spool fabrication company in Edmonton, Alberta. The framework consists of four components, namely the data source, data adapter, data analysis module, and main outputs. A workflow of the methodology developed to achieve the research objective is summarized in Figure 1. Each component is discussed in detail as follows:

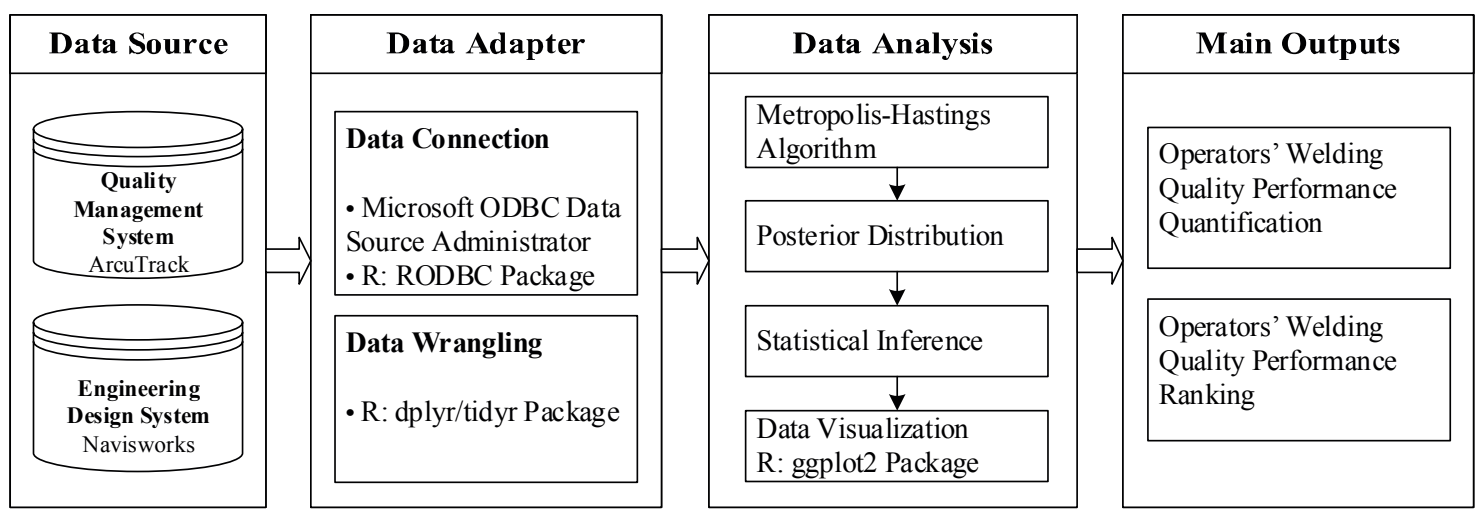

Figure 1: A workflow of the proposed methodology 


\subsection{Data Source}

\subsubsection{Quality Management System}

As required by the ISO 9000 standard, computer-based quality management systems have been widely implemented for quality management purposes within the construction industry (Chini and Valdez 2003). In practice, these systems are primarily used for quality inspection documentation and quality performance reporting purposes.

Data stored in the quality management system SQL server (ArcuTrack) of a pipe fabrication company in Edmonton, Canada were used to demonstrate the application of the proposed methodology. NDE inspection results and operator IDs for each weld were extracted and categorized as (1) no inspection performed, (2) inspected and passed, or (3) inspected and failed.

\subsubsection{Engineering Design System}

Building information modelling (BIM) is becoming increasingly implemented for its ability to improve information management of construction projects. Accordingly, detailed engineering models generated to facilitate quantity take-off, material information, and design specifications are accessible for many industrial construction projects. Pipe attributes, such as NPS, pipe schedule, material, and consequently, weld type, can be extracted from this information.

In the present study, pipe attributes were extracted from the engineering design system of the company studied. Here, pipe weld type was defined by pipe format (NPS, schedule, material, weld type). For example, pipe (40, 2, A, BW) represents butt welds with NPS of 40, schedule of 2 , and material A.

\subsection{Data Adapter}

A data adapter integrates information from various sources into a single, centralized database. This is particularly important for data that are collected from a variety of sources. In this case, a data adapter must be developed to transform raw data, through data connection and data wrangling, into compatible, interpretable formats.

Here, data was dispersed across quality management and engineering design systems. $\mathrm{R}$, a free software for statistical computing and graphics, was used to perform data connection and data wrangling tasks. For data connection, the RODBC package was used to connect to the SQL server of both the quality management and engineering design systems. The dplyr/tidyr package was used to perform data wrangling tasks, including data reshaping, grouping, and combining. The completed dataset was transformed into a table form, where each variable was saved in its own column and each observation was saved in its own row.

\subsection{Data Analysis Module}

Data analysis modules are comprised of a suite of algorithms that facilitate the establishment of metrics required by the framework. To accurately quantify operator welding performance, a distribution of this data should be determined using a data analysis module. The specialised algorithm and the generated metrics used in the present study are detailed as follows:

\subsubsection{Metropolis-Hastings Algorithm}

The Metropolis-Hastings algorithm is an MCMC-based method of generating random samples from a probability distribution. It was developed by Metropolis et al. (1953) and 
generalized by Hastings (1970). In the last decade, the Metropolis-Hastings algorithm has become increasingly popular within the statistical community for approximating distributions (Robert and Casella 2011).

Ji and AbouRizk (2017) developed a specialised Metropolis-Hastings algorithm for determining the posterior distribution and credible interval for fraction nonconforming. In the present study, this specialized algorithm was implemented to quantify operator welding quality performance.

The Metropolis-Hastings algorithm constructs a Markov chain of fraction nonconforming values for $\left\{p^{(1)}, p^{(2)}, p^{(3)}, \ldots, p^{(N)}\right\}$. The value $p^{(i+1)}$ is decided by proposing a random move conditional on the previous value $p^{(i)}$ and on the ratio of $\frac{P\left(p^{*} \mid X\right)}{P\left(p^{(i)} \mid X\right)}$. This acceptance ratio indicates the probably of the new proposed sample with respect to the current sample. The move is accepted if the new sample is more probable than the existing sample. Otherwise, the move is accepted with the acceptance probability, or the move is rejected. When these conditions are met, the Markov chain of parameter values will remain in the high-density region and will converge to the target distribution $P(p \mid X)$. As the sampling effort is concentrated in the area with higher posterior density, the time required for obtaining an acceptable convergence is typically shorter than those of other sampling techniques.

The logic of the specialised Metropolis-Hastings algorithm is illustrated in a step-bystep algorithmic form with the initial value $p^{(0)}$ and repeated for $i=1,2,3, \ldots, N$.

Step 1. Choose a new proposed value $p^{*}$, such that $p^{*}=p^{(i)}+\Delta p$, where $\Delta p \sim N(0, \sigma)$.

Step 2. Calculate the ratio $\rho=\min \left\{1, \frac{P\left(p^{*} \mid X\right)}{P\left(p^{(i)} \mid X\right)}\right\}$, where $P(p \mid X)$ is the posterior distribution.

Step 3. Sample $\mu \sim U_{[0,1]}$.

Step 4. If $\mu<\rho$

or else

$$
p^{(i+1)}=p^{*}
$$

$$
p^{(i+1)}=p^{(i)}
$$

Step 5. Return the values $\left\{p^{(1)}, p^{(2)}, p^{(3)}, \ldots, p^{(N)}\right\}$.

The draws $\left\{p^{(1)}, p^{(2)}, p^{(3)}, \ldots, p^{(N)}\right\}$ are regarded as a sample from the targeted distribution $P(p \mid X)$ only after the chain has passed the transient phase and the impact of the initial value can be ignored. After obtaining the fraction nonconforming values, $\left\{p^{(1)}, p^{(2)}, p^{(3)}, \ldots, p^{(N)}\right\}$, a frequency histogram plot is generated.

\subsubsection{Algorithm Validation}

Algorithm accuracy has been previously validated by comparing the derived analytical solution to that obtained using criteria of Mean Absolute Error (MAE) and Root Mean Square Error (RMSE). Readers are referred to Ji and AbouRizk (2017) for validation details and results.

\subsubsection{Simulation Example}

For illustration purposes, data for one welding operator with pipe (160, 2, A, BW) was used to demonstrate the algorithm. After connecting and processing the data, it was determined that 2 out of 57 welds failed inspection for operator A with pipe (160, 2, A, 
BW). By performing the specialised Metropolis-Hastings algorithm, the empirical posterior distribution was calculated. A frequency histogram plot and a box plot of the results are illustrated in Figure 2.

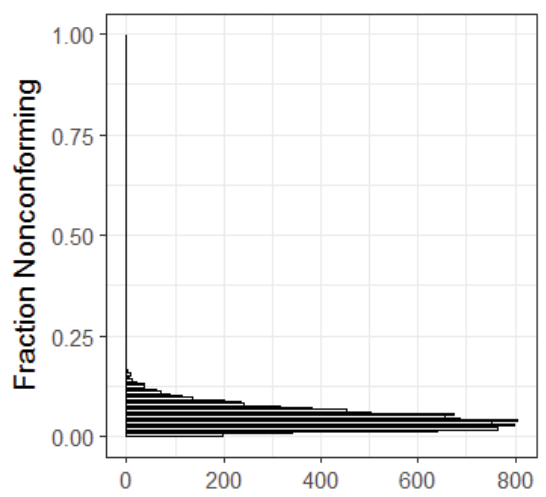

(a)

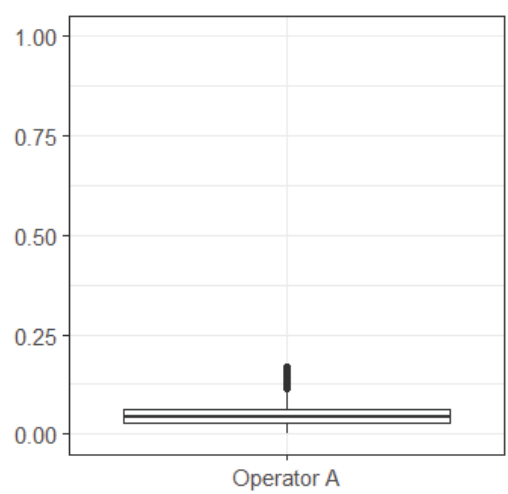

(b)

Figure 2: Empirical fraction nonconforming distribution for operator A with pipe (160, 2, A, BW): (a) frequency histogram plot; (b) boxplot.

A frequency histogram is a graphical representation of a frequency distribution of numerical data. A boxplot is a graphical representation of a five-number summary (Min, Q1, Median, Q3, Max). The upper whisker extends from the hinge to the highest value that is within $1.5{ }^{*}$ IQR of the hinge, where the IQR is the inter-quartile range or distance between the first and third quartiles. The lower whisker extends from the hinge to the lowest value within $1.5^{*}$ IQR of the hinge. Data beyond the end of the whiskers are outliers and are plotted as individual points.

As the algorithm is a numerical approximation to the true posterior distribution for the operators' welding quality performance, the numerical solution has a slight difference for each run of the proposed algorithm. Once the posterior distribution has been generated, statistics such as mean, median, variance, and quantiles can be easily obtained. The statistical software $\mathrm{R}$ is utilized to find the statistical summaries of interest. The statistical summaries of the example illustrated in Figure 2 are listed in Table 1.

Table 1: Statistical summary of operator A with Pipe (160, 2, A, BW).

\begin{tabular}{cccccccc}
\hline Statistical Summary & Mean & Variance & Min & Q1 & Median & Q3 & Max \\
\hline Fraction Nonconforming & 0.046 & 0.007 & 0.008 & 0.026 & 0.042 & 0.061 & 0.195 \\
\hline
\end{tabular}

\subsection{Main Outputs}

Practitioners are interested in identifying operators with exceptional quality performance for particular weld types. Therefore, the main outputs of the proposed framework include both the quantification and ranking of the operators' welding performance for each weld type. Outputs can be generated in a near real-time manner, which dramatically decreases the data interpretation load of decision makers.

In this section, data regarding pipe (STD, 2, A, BW), the most common work type, is used to demonstrate the main outputs of the proposed methodology. 17 operators, who have each had over 100 welds inspected, were selected. Table 2 lists the performance records and the five-number summary (Min, Q1, Median, Q3, and Max) of the welding quality performance with respect to fraction nonconforming of these operators. 
Implementing a Data-driven Simulation Method for Quantifying Pipe Welding Operator Quality

Performance

Consistent with Figure 2, the median value of their performance is sorted in descending order in Table 2. Operator IDs were reassigned to maintain employee anonymity.

Table 2: Statistical summary of operator welding performance of pipe

(STD, 2, A, BW).

\begin{tabular}{cccccccc}
\hline Operator ID & Inspected Welds & Repaired Welds & Min & Q1 & Median & Q3 & Max \\
\hline 1 & 175 & 25 & 0.069 & 0.128 & 0.147 & 0.166 & 0.243 \\
2 & 111 & 11 & 0.029 & 0.085 & 0.103 & 0.123 & 0.225 \\
3 & 139 & 13 & 0.036 & 0.080 & 0.096 & 0.113 & 0.193 \\
4 & 307 & 27 & 0.046 & 0.078 & 0.089 & 0.099 & 0.157 \\
5 & 100 & 8 & 0.026 & 0.065 & 0.081 & 0.100 & 0.223 \\
6 & 207 & 16 & 0.028 & 0.068 & 0.080 & 0.093 & 0.159 \\
7 & 104 & 7 & 0.009 & 0.055 & 0.070 & 0.087 & 0.196 \\
8 & 119 & 8 & 0.015 & 0.054 & 0.069 & 0.086 & 0.182 \\
9 & 175 & 11 & 0.023 & 0.053 & 0.064 & 0.076 & 0.138 \\
10 & 175 & 9 & 0.017 & 0.040 & 0.052 & 0.067 & 0.133 \\
11 & 120 & 6 & 0.011 & 0.038 & 0.052 & 0.064 & 0.155 \\
12 & 316 & 16 & 0.020 & 0.043 & 0.051 & 0.059 & 0.097 \\
13 & 208 & 9 & 0.009 & 0.035 & 0.043 & 0.054 & 0.110 \\
14 & 123 & 5 & 0.006 & 0.033 & 0.043 & 0.057 & 0.119 \\
15 & 147 & 5 & 0.005 & 0.025 & 0.035 & 0.047 & 0.097 \\
16 & 264 & 9 & 0.013 & 0.029 & 0.035 & 0.043 & 0.080 \\
17 & 355 & 11 & 0.008 & 0.027 & 0.031 & 0.038 & 0.072 \\
\hline
\end{tabular}

To visually represent and compare operator welding performance, a side-by-side boxplot was generated, as in Figure 2, and is shown in Figure 3. Box width is inversely proportional to the stability of operator quality performance, where a narrower box is indicative of an operator with stable quality performance for the indicated type of weld. Operators with lower ranks have smaller fraction nonconforming (i.e., better performance). The dashed line represents the overall averaged welding quality performance of the 17 operators (fraction nonconforming=0.062). The range of the median is relatively wide (0.031 to 0.147$)$, indicating operator performance varies considerably for the same type of welding work. Although root cause analysis may identify factors affecting operator welding performance, it is beyond the scope of this study.

From this visualized output (Figure 3), practitioners can (1) infer operators' skill level for a specified type of work; (2) identify operators who consistently produce high-quality welds; and (3) support future decision-making processes. These results can have considerable, positive impacts on company quality and productivity performance. Potential applications of this research are discussed in the following section. 


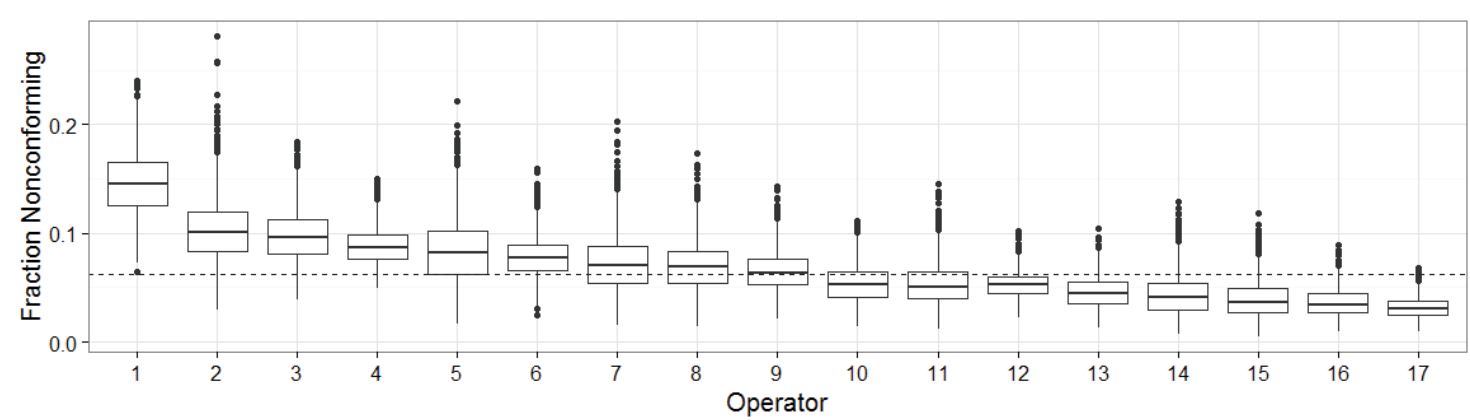

Figure 3: Sorted side-by-side boxplot of operators' welding performance with pipe (STD, 2, A, BW).

\section{Potential APPLICATIONS}

To strategically improve fabrication companies' competitiveness and reputation within the construction market, operators with exceptional welding quality performance should be effectively and efficiently utilized. Here, three potential applications of the proposed research outcome are identified from the perspectives of (1) production planning; (2) employee training; and (3) strategic recruiting. These three potential applications may lead to considerable improvements in pipe welding quality, while potentially reducing cost and schedule overruns induced by poor quality welds.

\subsection{Production Planning}

Operator welding quality performance varies between weld types. Development and implementation of an optimal, performance-driven production planning optimization engine, which would allocate welding work to operators with highest performance quality for that particular work/weld type, could directly improve overall welding quality performance.

\subsection{Employee Training}

Following the implementation of the proposed methodology, practitioners will be able to determine which operators are most proficient for each weld type. These operators should be invited to demonstrate their operation processes and share their professional knowledge for employee training purposes. Companies may also standardize the operation processes as per the high-performance welders' operation processes. Enhanced training programs are crucial for improving the welding quality performance of all operators.

\subsection{Strategic Recruiting}

Once high-performance operators are identified, their profiles can be examined to identify critical success factors common to this group. Data mining techniques may be implemented to facilitate complex analytical processes. Human resource teams may strategically conduct data-driven recruiting to hire qualified welding operators who are characterized by these success factors.

\section{CONCLUSIONS}

The construction industry is a labour-intensive industry whose productivity is heavily dependent on operator performance. This paper proposes an MCMC-based simulation 
approach to determine the posterior distribution for quantifying operator welding quality performance for a specific work type. Real quality management data and engineering design data from a pipe fabrication company were extracted to demonstrate the applicability of the proposed approach. Operator welding quality performances were represented by posterior distributions to incorporate uncertainty. Statistics such as mean, median, and quantiles were easily obtained to support decision-making processes. Practitioners can implement this approach to (1) infer operators' skill level for a specified type of work; (2) identify operators who consistently produce high-quality welds; and (3) support future decision-making processes and improve production planning, employee training, and strategic recruiting.

For the future work, this research provides inputs for considering operators' welding quality performance level in welding processes simulation. Also, once the fraction nonconforming is quantified at a detailed level, the quality-induced rework cost can be estimated in a more accurate and reliable way.

\section{ACKNOWLEDGEMENTS}

This research is funded by NSERC and Falcon Fabricators \& Modular Builders, Ltd. The authors would like to acknowledge Rob Reid, Doug McCarthy, Jason Davio, and Christian Jukna for sharing their knowledge and expertise of pipe fabrication quality management.

\section{REFERENCES}

American Society of Mechanical Engineers. (2005). Process Piping: ASME Code for Pressure Piping, B31. American Society of Mechanical Engineers.

Chini, A. R., and Valdez, H. E. (2003). ISO 9000 and the US construction industry. Journal of management in engineering, 19(2), 69-77.

Hastings, W. K. (1970). Monte Carlo sampling methods using Markov chains and their applications. Biometrika, 57(1), 97-109.

Ji, W., and AbouRizk, S. M. (2016). A Bayesian inference based simulation approach for estimating fraction nonconforming of pipe spool welding processes. In Proceedings of the 2016 Winter Simulation Conference (WSC). IEEE.

Ji, W., and AbouRizk, S. M. (2017). Credible interval estimation for fraction nonconforming: analytical and numerical solutions. Automation in Construction (in revision).

Montgomery, D. C. (2007). Introduction to statistical quality control. John Wiley \& Sons.

Metropolis, N., Rosenbluth, A. W., Rosenbluth, M. N., Teller, A. H., and Teller, E. (1953). Equation of state calculations by fast computing machines. The Journal of Chemical Physics, 21(6), 1087-1092.

Robert, C., and Casella, G. (2011). A short history of Markov Chain Monte Carlo: subjective recollections from incomplete data. Statistical Science, 102-115.

Weiss, N. (2013). Introductory Statistics. Pearson.

Wang, P., Mohamed, Y., Abourizk, S.M., and Rawa, A.T. (2009). Flow production of pipe spool fabrication: Simulation to support implementation of lean technique. Journal of Construction Engineering and Management,135(10), pp.1027-1038.

Weaver, B. P., and Hamada, M. S. (2016). Quality quandaries: A gentle introduction to Bayesian statistics. Quality Engineering, 28(4), 508-514. 